\section{Stromal-immune cell crosstalk deciphered in Dupuytren disease}

Fibrotic diseases are some of the most challenging conditions to treat owing to a lack of effective therapies. Drug development for such conditions is hampered by a lack of suitable animal models and a poor understanding of the early stages of the pathogenic process. A new study is improving this understanding by revealing details of the cellular environment in Dupuytren disease, a fibrotic disease of the hand.

"Localized fibrotic conditions such as Dupuytren disease are relatively neglected and yet provide a unique opportunity to study human fibrosis as they can be diagnosed early and there is an abundant supply of human tissue following surgical excision," explains corresponding author Jagdeep Nanchahal. "Our study provides a detailed single-cell analysis of the immune landscape in Dupuytren disease and identifies a reciprocal pathogenic signalling circuit between stromal and immune cells."
Previous work from this group had revealed that low concentrations of TNF were present in Dupuytren disease nodules and were necessary for the activation of myofibroblasts, which led to ongoing phase II clinical trials of TNF inhibitors for this disease. In their new study, Nanchahal and colleagues combined single-cell RNA analysis with flow cytometry and in vitro functional studies to decipher which cells produce TNF

"We demonstrated that M2 macrophages and mast cells are key cellular sources of TNF that promotes myofibroblast development," says Nanchahal. "TNF acts via the inducible TNFR2 receptor and stimulates IL-33 secretion by myofibroblasts. In turn, this IL-33 acts as a potent stimulus for TNF production from the immune cells."

Blocking TNFR2 and IL-33 inhibited the expression of within fibrotic tissue.

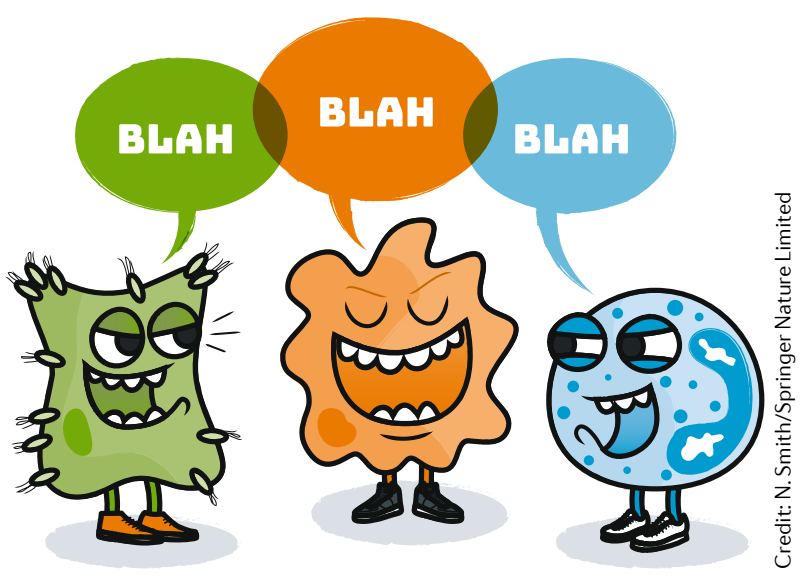

pro-fibrotic genes in myofibroblasts from patients with Dupuytren disease, and also reduced the fibrotic function (contractility) of the cells. By contrast, blocking the widely expressed TNF receptor TNFR1 did not reduce the expression of pro-fibrotic genes in these cells, suggesting that selectively targeting TNFR2 might be a more effective therapeutic strategy than targeting systemic TNF.

Joanna Clarke

ORIGINAL ARTICLE Izadi, D. et al. Identification of TNFR2 and IL-33 as therapeutic targets in localized fibrosis. Sci. Adv. 5, eaay0370 (2019)

\section{EXPERIMENTAL ARTHRITIS}

\section{Pro-resolving receptor protects against arthritis}

$\mathrm{N}-3$ docosapentaenoic acid-derived resolvin D5 (RvD5 $\left.5_{n-3 \text { DPA }}\right)$ is a specialized pro-resolving mediator that is important for the resolution of inflammation. Previous work has highlighted the protective role of RvD5 $5_{n-3 \text { DPA }}$ in limiting inflammation and joint damage in arthritis. A new study sheds further light on this axis, and identifies GPR101 as the receptor responsible for mediating the pro-resolving functions of RvD5 $5_{n-3}$ DPA.

"We employed a screening approach to identify candidate G-protein-coupled receptors that might be activated by RvD5 ${ }_{n-3 \text { DPA, }}$, reports Jesmond Dalli, corresponding author on the new study. The researchers found that one candidate - GPR101-bound $\mathrm{RvD5}_{\mathrm{n}-3 \mathrm{DPA}}$ with a high selectivity $\left(K_{\mathrm{d}} 6.9 \mathrm{nM}\right)$ and high stereospecificity. Furthermore, this receptor was expressed on human peripheral leukocytes (neutrophils and monocytes) in the blood.

Addition of RvD5 $5_{n-3 D P A}$ to human macrophages in vitro led to a dosedependent increase in phagocytosis and efferocytosis whereas small interfering RNA (siRNA)-mediated knockdown of GPR101 reversed these effects. Similarly, treatment of human neutrophils with an antibody targeting GPR101 also limited the dose-dependent decrease in chemotaxis and migration

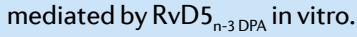

In mice with $\mathrm{K} / \mathrm{B} \times \mathrm{N}$ serum transferinduced arthritis, treatment with $\mathrm{RvD5}_{\mathrm{n}-3 \mathrm{DPA}}$ led to improved clinical scores (compared with treatment with vehicle), and protected the mice against weight loss and joint oedema. Notably, in vivo siRNA-mediated knockdown of GPR101 reversed the anti-arthritic effects of RvD5 $5_{n-3 D P A}$.

"The identification of GPR101 as a pro-resolving receptor provides new understanding as to how chronic inflammatory conditions might occur as well as targets for new therapeutics to treat inflammatory conditions including rheumatoid arthritis," says Dalli.

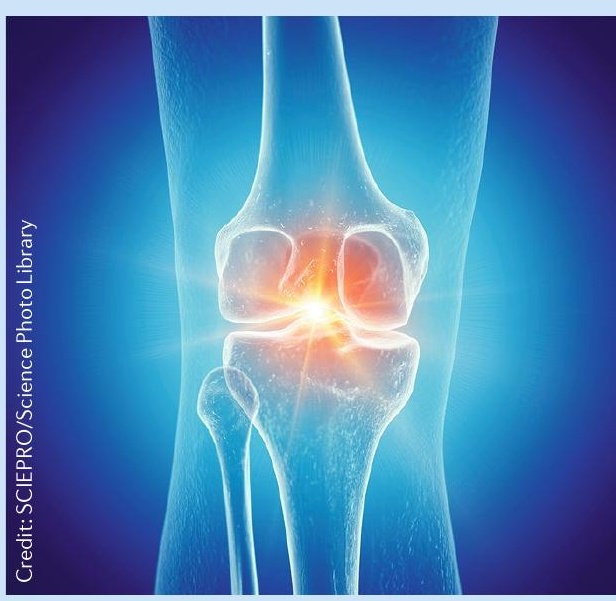

The researchers plan to investigate the pathways that are promoted downstream of RvD5 ${ }_{n-3}$ DPA $-G P R 101$ engagement, and whether these pathways are altered in disease. "Such investigations will enable us to establish whether disruptions in this axis might contribute to inflammatory disorders," remarks Dalli.

Jessica McHugh

ORIGINAL ARTICLE Flak, M. B. et al. GPR101

mediates the pro-resolving actions of $\mathrm{RvD} 5_{n-3 \mathrm{DP}}$ in arthritis and infections.J. Clin. Invest. https:// doi.org/10.1172/JCl131609 (2019) 